Y. Abe

Nagoya Math. J.

Vol. 116 (1989), 17-24

\title{
HOMOGENEOUS LINE BUNDLES OVER A TOROIDAL GROUP
}

\author{
YUKITAKA ABE
}

\section{§. Introduction}

A connected complex Lie group without non-constant holomorphic functions is called a toroidal group ([5]) or an $(H, C)$-group ([9]). Let $X$ be an $n$-dimensional toroidal group. Since a toroidal group is commutative ([5], [9] and [10]), $X$ is isomorphic to the quotient group $C^{n} / \Gamma$ by a lattice of $C^{n}$. A complex torus is a compact toroidal group. Cousin first studied a non-compact toroidal group ([2]).

Let $L$ be a holomorphic line bundle over $X . \quad L$ is said to be homogeneous if $T_{x}^{*} L$ is isomorphic to $L$ for all $x \in X$, where $T_{x}$ is the translation defined by $x \in X$. It is well-known that if $X$ is a complex torus, then the following assertions are equivalent:

(1) $L$ is topologically trivial.

(2) $L$ is given by a representation of $\Gamma$.

(3) $L$ is homogeneous.

But this is not always true for a toroidal group. Vogt showed in [11] that every topologically trivial holomorphic line bundle over $X$ is homogeneous if and only if $\operatorname{dim} H^{1}(X, \mathcal{O})<\infty$ ([6]). The cohomology groups $H^{p}(X, \mathcal{O})$ were classified by Kazama [3] and Kazama-Umeno [4].

In this paper we shall show the equivalence of conditions (2) and (3). In the case that $X$ is a complex torus, a similar equivalence was proved for a vector bundle ([7] and [8]). We state our theorem.

Theorem. Let $X=C^{n} / \Gamma$ be a toroidal group. Then every homogeneous line bundle over $X$ is given by a 1-dimensional representation of $\Gamma$.

The converse of the above theorem is easily seen by the definitions ([11, Proposition 6]). We shall prove the theorem by virtue of the following proposition.

Proposition. Every homogeneous line bundle over a toroidal group is topologically trivial.

Received July 27, 1987. 


\section{$\S 1$. Preliminaries}

We state some results concerning toroidal groups and fix the notations used in this paper.

If $X=C^{n} / \Gamma$ is a toroidal group, then the rank of $\Gamma$ is $n+m, 0<m$ $\leqq n, \quad$ Let $p^{1}=\left(p_{11}, \cdots, p_{n, 1}\right), \cdots, p^{n+m}=\left(p_{1, n+m}, \cdots, p_{n, n+m}\right) \in C^{n}$ be generators of $\Gamma$. The $n \times(n+m)$ matrix

$$
P=\left({ }^{t} p^{1}, \cdots,{ }^{t} p^{n+m}\right)
$$

is called a period matrix of $\Gamma$. We may assume by Proposition 2 in [11] that $\Gamma$ has a period matrix $P$ as follows

$$
P=\left(\begin{array}{cc}
0 & T \\
I_{n-m} & R
\end{array}\right),
$$

where $I_{n-m}$ is the $(n-m) \times(n-m)$ unit matrix, $T$ is a period matrix of an $m$-dimensional complex torus and $R$ is a real $(n-m) \times 2 m$ matrix with

$$
\sigma R \neq 0 \quad \bmod Z^{2 m} \quad \text { for all } \sigma \in Z^{n-m} \backslash\{0\} .
$$

Let $\boldsymbol{R}_{\Gamma}^{n+m}$ be the real-linear subspace of $\boldsymbol{C}^{n}$ spanned by $\Gamma$. We denote by $\boldsymbol{C}_{\Gamma}^{m}$ the maximal complex-linear subspace contained in $\boldsymbol{R}_{\Gamma}^{n+m}$. When a period matrix $P$ of $\Gamma$ has the form as (1.1), $C_{\Gamma}^{m}$ is the space of the first $m$ variables. Then we take the coordinates of $C^{n}=C_{\Gamma}^{m} \times C^{n-m}$ as $(z, w)$ with $z \in C_{\Gamma}^{m}, w \in C^{n-m}$.

We refer the reader to [11] for the definitions of factors of automorphy and summands of automorphy.

Lemma 1 ([11, Proposition 8]). Let $X=C^{n} / \Gamma$ be a toroidal group. Then every summand of automorphy $b: \Gamma \times C^{n} \rightarrow C$ is equivalent to $a$ summand of automorphy $a: \Gamma \times C^{n} \rightarrow C$ with the following properties:

a) $a(\gamma ; z, w)=a(\gamma, w)$ for all $\gamma \in \Gamma$.

b) $a(\gamma ; z, w)=0$ for $\gamma \in\left(\begin{array}{ll}0 & Z^{n-m}\end{array}\right)$.

c) For all $\gamma \in \Gamma$ the holomorphic function $a_{r}(w):=a(\gamma, w)$ is $Z^{n-m_{-}}$ periodic.

A homomorphism $\alpha: \Gamma \rightarrow C^{*}$ is called a (1-dimensional) representation of $\Gamma$. Two representations $\alpha$ and $\beta$ of $\Gamma$ are equivalent if there exists a holomorphic function $g: C^{n} \rightarrow C^{*}$ such that

$$
g(x+\gamma) \alpha(\gamma) g(x)^{-1}=\beta(\gamma)
$$


for all $\gamma \in \Gamma$ and $x=(z, w) \in C^{n}$.

Lemma 2. Let $X=C^{n} / \Gamma$ be a toroidal group and let $\alpha: \Gamma \rightarrow C_{1}^{\times}=$ $\{\zeta \in C ;|\zeta|=1\}$ be a homomorphism. If $\alpha$ is equivalent to the constant map 1 , then there exists a $C$-linear form $L$ on $C^{n}$ depending only on $w$ such that

$$
\alpha(\gamma)=e(L(\gamma)) \quad \text { for all } \gamma \in \Gamma,
$$

where $\boldsymbol{e}(x)=\exp (2 \pi \sqrt{-1} x)$.

Proof. By the assumption, there exists a holomorphic function $g$ : $C^{n} \rightarrow C^{*}$ such that

$$
g(x+\gamma) \alpha(\gamma) g(x)^{-1}=1 \quad \text { for all } \gamma \in \Gamma \text { and } x \in C^{n} .
$$

We have a holomorphic function $h: C^{n} \rightarrow C$ with $g(x)=\boldsymbol{e}(h(x))$. All first order derivatives of $h$ are $\Gamma$-periodic by (1.3). Then we can write $h(x)=$ - $\mathscr{L}(x)+c$, where $\mathscr{L}(x)$ is a $C$-linear form on $C^{n}$ and $c$ is a complex number. Using (1.3) again, we have $\alpha(\gamma)=\boldsymbol{e}(\mathscr{L}(\gamma))$. Since $|\alpha(\gamma)|=1$ for all $\gamma \in \Gamma, L$ is real-valued on $\boldsymbol{R}_{\Gamma}^{n+m}$. Then $L$ is constant on $C_{\Gamma}^{m}$.

A factor of automorphy $\alpha(\gamma ; z, w)$ is called a theta factor if it is expressed by a linear polynomial $\ell_{r}(z, w)$ on $(z, w)$ as $\alpha(\gamma ; z, w)=\boldsymbol{e}\left(\ell_{r}(z, w)\right)$.

Lemma 3 ([5]). Let $\rho(\gamma ; z, w)$ be a theta factor for $\Gamma$ on $C^{n}$. Then there exist a hermitian form $\mathscr{H}$ on $C^{n} \times C^{n}$ with $\mathscr{A}:=\operatorname{Im} \mathscr{H} Z$-valued on $\Gamma \times \Gamma, a C$-bilinear symmetric form $\mathscr{Q}, a C$-linear form $\mathscr{L}$ and a semicharacter $\psi$ of $\Gamma$ associated with $\left.\mathscr{A}\right|_{\Gamma \times \Gamma}$ such that

$$
\rho(\gamma ; z, w)=\psi(\gamma) \boldsymbol{e}\left[\frac{1}{2 \sqrt{-1}}(\mathscr{H}+\mathscr{2})(\gamma ; z, w)+\frac{1}{4 \sqrt{-1}}(\mathscr{H}+\mathscr{2})(\gamma, \gamma)+\mathscr{L}(\gamma)\right]
$$

for all $\gamma \in \Gamma$ and $(z, w) \in C^{n}$. We say that $\rho$ is of type $(\mathscr{H}, \psi, \mathscr{Q}, \mathscr{L})$ when it has an expression as the above.

Remark. If rank $\Gamma=2 n$, then $\rho$ is of the unique type. But in general, a type of $\rho$ is not uniquely decided. Let $\boldsymbol{R}_{\Gamma}^{n+m}=C_{\Gamma}^{m} \oplus V$, where $V$ is a real-linear subspace of $\boldsymbol{R}_{\Gamma}^{n+m}$. Then $\boldsymbol{C}^{n}=\boldsymbol{C}_{\Gamma}^{m} \oplus V \oplus \sqrt{-1} V$. A hermitian form $\mathscr{H}$ changes according to the choice of $\left.\mathscr{A}\right|_{V \times \sqrt{-1} V}$. We may assume that $\left.\mathscr{A}\right|_{V \times \sqrt{-1} V}=0$.

\section{§. Proof of the proposition}

Let $L$ be a homogeneous line bundle over a toroidal group $X=C^{n} / \Gamma$. We may assume by a result of $\operatorname{Vogt~([12],~see~also~[1])~that~} L=L_{\alpha} \otimes L_{\rho}$, 
where $L_{\alpha}$ is a topologically trivial holomorphic line bundle given by a factor of automorphy $\alpha$ and $L_{\rho}$ is a theta bundle given by a theta factor $\rho$. Furthermore we may assume that $\rho$ is reduced, i.e. $\rho$ is of type $(\mathscr{H}, \psi)$ $=(\mathscr{H}, \psi, 0,0)$, and $\alpha$ has the properties in Lemma 1.

Let $\pi: C^{n} \rightarrow X$ be the projection. Take any $x^{*}=\left(x_{1}^{*}, x_{2}^{*}\right) \in C_{\Gamma}^{m} \times Z^{n-m}$, and set $x=\pi\left(x^{*}\right)$. The pull-back $T_{x}^{*} L$ of $L$ by a translation $T_{x}$ is given by a factor of automorphy $\alpha\left(\gamma, w-x_{2}^{*}\right) \rho\left(\gamma ; z-x_{1}^{*}, w-x_{2}^{*}\right)$. Since $\alpha(\gamma, w)$ is $Z^{n-m}$-periodic, we have $T_{x}^{*} L_{\alpha}=L_{\alpha}$. Then $T_{x}^{*} L_{\rho} \cong L_{\rho}$. We set $a:=-x^{*}$ and $\rho_{1}(\gamma ; z, w):=\rho\left(\gamma ; z-x_{1}^{*}, w-x_{2}^{*}\right)$. Then $\rho_{1}$ is of type $\left(\mathscr{H}, \psi_{1}, 0, \mathscr{L}_{1}\right)$, where

$$
\begin{aligned}
& \psi_{1}(\gamma):=\psi(\gamma) \boldsymbol{e}(-\mathscr{A}(a, \gamma)), \\
& \mathscr{L}_{1}(z, w):=\frac{1}{2 \sqrt{-1}} \mathscr{H}(a ; z, w) .
\end{aligned}
$$

We define a homomorphism $\beta: \Gamma \rightarrow C_{1}^{\times}$by

$$
\beta(\gamma):=\psi(\gamma) \psi_{1}(\gamma)^{-1}=e(\mathscr{A}(a, \gamma)) .
$$

Since $\rho \cdot \rho_{1}^{-1}$ is equivalent to $1, \beta$ is also equivalent to 1 . By Lemma 2 there exists a $\boldsymbol{C}$-linear form $\mathscr{L}$ on $\boldsymbol{C}^{n}$ depending only on $w$ such that

$$
\beta(\gamma)=\boldsymbol{e}(\mathscr{L}(\gamma)) \quad \text { for all } \gamma \in \Gamma .
$$

It follows immediately from the above equality that

$$
\mathscr{A}(a, x)=\mathscr{L}(x) \quad \text { for all } x \in \boldsymbol{R}_{\Gamma}^{n+m} .
$$

Since $a \in C_{\Gamma}^{m} \times Z^{n-m}$ is arbitrary, have

$$
\mathscr{A}(x, y)=0 \quad \text { for all } x \in \boldsymbol{R}_{\Gamma}^{n+m} \text { and } y \in \boldsymbol{C}_{\Gamma}^{m} .
$$

By Remark below Lemma 3 we may assume that $\left.\mathscr{A}\right|_{V \times \sqrt{-1} V}=0$. Then we have

$$
\left.\mathscr{A}\right|_{C_{\Gamma}^{m} \times C^{n}}=0 \text { and }\left.\quad \mathscr{A}\right|_{C^{n} \times C_{\Gamma}^{m}}=0,
$$

because $\mathscr{A}$ is the imaginary part of a hermitian form $\mathscr{H}$. By (2.1) a hermitian form $\mathscr{H}$ is regarded as a hermitian form on $C^{n-m} \times C^{n-m}$.

We set $\left(I_{n-m} R\right)=\left({ }^{t} e_{1}, \cdots,{ }^{t} e_{n-m},{ }^{t} r_{1}, \cdots,{ }^{t} r_{2 m}\right)$ in the period matrix (1.1). Every $r_{k}$ is represented as

$$
r_{k}=\sum_{j=1}^{n-m} r_{j, k} e_{j}, \quad r_{j, k} \in \boldsymbol{R} .
$$

For any $i$ and $k$ we have 


$$
\mathscr{A}\left(e_{i}, r_{k}\right)=\sum_{j=1}^{n-m} r_{j, k} \mathscr{A}\left(e_{i}, e_{j}\right) \in Z
$$

Since $X=C^{n} / \Gamma$ is a toroidal group, we obtain by (1.2) that

$$
\mathscr{A}\left(e_{i}, e_{j}\right)=0 \quad \text { for all } i, j=1, \cdots, n-m \text {. }
$$

By (2.1) and (2.2) we conclude

$$
\mathscr{A}=0 \quad \text { on } \boldsymbol{C}^{n} \times C^{n},
$$

hence $\mathscr{H}=0$ on $\boldsymbol{C}^{n} \times \boldsymbol{C}^{n}$. This means that $L_{\rho}$ is given by a representation of $\Gamma$, therefore $L_{\rho}$ is topologically trivial.

\section{$\S 3$. Proof of the theorem}

Let $L$ be a homogeneous line bundle over a toroidal group $X=C^{n} / \Gamma$. By Proposition $L$ is topologically trivial. Then $L$ is given by a factor of automorphy $\alpha(\gamma, w)=\exp (a(\gamma, w))$, where a summand of automorphy $a(\gamma, w)$ has the properties in Lemma 1 . Since $L$ is homogeneous, $a(\gamma, w+x)$ and $a(\gamma, w)$ are equivalent for all $x \in C^{n-m}$. That is, there exist a holomorphic function $g_{x}: C^{n} \rightarrow C$ and a homomorphism $n_{x}: \Gamma \rightarrow Z$ for any $x$ such that

$$
g_{x}\left(z+\gamma_{1}, w+\gamma_{2}\right)-g_{x}(z, w)=a(\gamma, w+x)-a(\gamma, w)+2 \pi \sqrt{-1} n_{x}(\gamma)
$$

for all $\gamma=\left(\gamma_{1}, \gamma_{2}\right) \in \Gamma$ and $(z, w) \in C^{n}$. We see by (3.1) that all first order derivatives of $g_{x}$ with respect to $z$ are $\Gamma$-periodic. Then $g_{x}$ is expressed as

$$
g_{x}(z, w)=\ell_{x}(z)+h_{x}(w),
$$

where $\ell_{x}(z)$ is a $C$-linear form on $C_{\Gamma}^{m}$ and $h_{x}(w)$ is a holomorphic function on $\boldsymbol{C}^{n-m}$. By (3.1) it holds that

$$
\begin{aligned}
h_{x}\left(w+\gamma_{2}\right)-h_{x}(w) & =a(\gamma, w+x)-a(\gamma, w)+2 \pi \sqrt{-1} n_{x}(\gamma)-\ell_{x}\left(\gamma_{1}\right) \\
& =a(\gamma, w+x)-a(\gamma, w)+c_{x}(\gamma)
\end{aligned}
$$

for all $\gamma \in \Gamma$ and $w \in C^{n-m}$, where we set $c_{x}(\gamma)=2 \pi \sqrt{-1} n_{x}(\gamma)-\ell_{x}\left(\gamma_{1}\right)$.

Let $p^{j}=\left(p_{1}^{j}, p_{2}^{j}\right) \in C_{\Gamma}^{m} \times C^{n-m}$. We define a $C$-linear form $\mathscr{L}_{x}(w)$ on $C^{n-m}$ by

$$
\mathscr{L}_{x}(w):=\sum_{j=1}^{n-m} c_{x}\left(p^{j}\right) w_{j}
$$

Putting $\tilde{g}_{x}(w):=h_{x}(w)-\mathscr{L}_{x}(w)$, we have by (3.2) that

$$
\tilde{g}_{x}\left(w+\gamma_{2}\right)-\tilde{g}_{x}(w)=a(\gamma, w+x)-a(\gamma, w)+c_{x}(\gamma)-\mathscr{L}_{x}\left(\gamma_{2}\right)
$$


for all $\gamma \in \Gamma$ and $w \in C^{n-m}$. We set newly $g_{x}(w)=\tilde{g}_{x}(w)$ and $c_{x}(\gamma)=c_{x}(\gamma)$ $-\mathscr{L}_{x}\left(\gamma_{2}\right)$. Then we have

$$
g_{x}\left(w+\gamma_{2}\right)-g_{x}(w)=a(\gamma, w+x)-a(\gamma, w)+c_{x}(\gamma)
$$

for all $\gamma \in \Gamma$ and $w \in C^{n-m}$, where $c_{x}(\gamma)=0$ for $\gamma \in\left(0 \quad Z^{n-m}\right)$ and $g_{x}(w)$ is a $Z^{n-m}$-periodic holomorphic function on $C^{n-m}$.

We set $\left(I_{n-m} R\right)=\left({ }^{t} s_{1}, \cdots,{ }^{t} s_{n+m}\right)$, i.e. $s_{j}=p_{2}^{j}$ and define

$$
b_{x}^{j}(w):=a\left(p^{j}, w+x\right)-a\left(p^{j}, w\right)+c_{x}\left(p^{j}\right) .
$$

Then $b_{x}^{j}(w)$ is a $Z^{n-m}$-periodic holomorphic function on $\boldsymbol{C}^{n-m}$. We obtain by $\left(3.1^{\prime}\right)$ that

$$
g_{x}\left(w+s_{j}\right)-g_{x}(w)=b_{x}^{j}(w), \quad j=1, \cdots, n+m .
$$

We put

$$
\begin{aligned}
a\left(p^{j}, w\right) & =\sum_{\sigma \in \mathbf{Z}^{n-m}} a_{j, \sigma} \boldsymbol{e}\left(\sigma^{t} w\right), \\
b_{x}^{j}(w) & =\sum_{\sigma \in Z^{n-m}} b_{x, \sigma}^{j} \boldsymbol{e}\left(\sigma^{t} w\right)
\end{aligned}
$$

and

$$
g_{x}(w)=\sum_{\sigma \in \bar{Z}^{n-m}} g_{x, \sigma} \boldsymbol{e}\left(\sigma^{t} w\right)
$$

Since $g_{x}(w)$ is a solution of the system of difference equations (3.3), we have

$$
b_{x, 0}^{j}=c_{x}\left(p^{j}\right)=0
$$

and

$$
g_{x, \sigma}=\frac{b_{x, \sigma}^{j}}{\boldsymbol{e}\left(\sigma^{t} s_{j}\right)-1}, \quad \sigma \neq 0
$$

for $j$ with $\sigma^{t} s_{j} \notin Z$ ([11, Lemma 2]). The system of difference equations (3.3) is independent of $g_{x, 0}$. So we may assume that $g_{x, 0}=0$. It follows from the definition of $b_{x}^{j}$ that

$$
g_{x, \sigma}=a_{j, \sigma} \frac{\boldsymbol{e}\left(\sigma^{t} x\right)-1}{\boldsymbol{e}\left(\sigma^{t} s_{j}\right)-1}, \quad \sigma \neq 0 .
$$

For any $\gamma \in \Gamma$ we have

$$
\boldsymbol{e}\left(\sigma^{t}\left(x+\gamma_{2}\right)\right)-1=\boldsymbol{e}\left(\sigma^{t} \gamma_{2}\right)\left(\boldsymbol{e}\left(\sigma^{t} x\right)-1\right)+\boldsymbol{e}\left(\sigma^{t} \gamma_{2}\right)-1 .
$$

Using $\left(3.1^{\prime}\right),(3.4)$ and the above equality, we get

$$
g_{x+\gamma_{2}}(w)-g_{x}(w)=a(\gamma, w+x)-a(\gamma, w)+g_{\gamma_{2}}(w)
$$


for all $\gamma \in \Gamma$ and $w \in C^{n-m}$.

The series $\sum_{\sigma \in Z^{n-m}} g_{x, \sigma}$ is absolutely convergent at each point $x \in C^{n-m}$. We shall show that this series is uniformly absolutely convergent in the wider sense on $C^{n-m}$. Let

$$
A_{\sigma}:= \begin{cases}\frac{a_{j, \sigma}}{\boldsymbol{e}\left(\sigma^{t} s_{j}\right)-1} & \text { if } \sigma \neq 0 \\ 0 & \text { if } \sigma=0 .\end{cases}
$$

Then

$$
g_{x, \sigma}=A_{\sigma}\left(e\left(\sigma^{t} x\right)-1\right) \quad \text { for } \sigma \neq 0 .
$$

It suffices to show that $\sum_{\sigma \in Z^{n-m}} A_{\sigma} X^{\sigma}$ is uniformly absolutely convergent in the wider sense of $C^{n-m}$. Now we set

$$
r_{\sigma}(x):=\exp \left(-2 \pi \sigma^{t} \operatorname{Im} x\right) .
$$

Then we have

$$
\left|g_{x, \sigma}\right| \geqq\left|A_{\sigma}\right|\left|r_{\sigma}(x)-1\right| \text {. }
$$

We can write $r_{\sigma}(x)=r_{1}\left(x_{1}\right)^{\sigma_{1} \cdots r_{n-m}}\left(x_{n-m}\right)^{\sigma_{n-m}}$, where $r_{i}\left(x_{i}\right):=\exp \left(-2 \pi \operatorname{Im} x_{i}\right)$, $i=1, \cdots, n-m$. There exists a positive number $C$ such that for sufficiently large $r_{1}\left(x_{1}\right), \cdots, r_{n-m}\left(x_{n-m}\right)$

$$
\left|r_{\sigma}(x)-1\right| \geqq C r_{1}\left(x_{1}\right)^{\sigma_{1} \ldots r_{n-m}}\left(x_{n-m}\right)^{\sigma_{n-m}}
$$

for all $\sigma_{1}>0, \cdots, \sigma_{n-m}>0$. Thus we have

$$
\begin{aligned}
& \sum_{\sigma_{1} \geqq 0, \ldots, \sigma_{n-m} \geqq 0}\left|A_{\sigma}\right|\left|r_{\sigma}(x)-1\right| \\
& \geqq C \sum_{\sigma_{1}>0, \cdots, \sigma_{n-m} \geqq 0}\left|A_{\sigma}\right| r_{1}\left(x_{1}\right)^{\sigma_{1} \ldots r_{n-m}}\left(x_{n-m}\right)^{\sigma_{n-m}} .
\end{aligned}
$$

This implies that the series $\sum_{\sigma_{1} \geqq 0, \ldots, \sigma_{n-m} \geqq 0} A_{\sigma} X^{\sigma}$ is absolutely convergent in the wider sense on $C^{n-m}$. Also we have

$$
\begin{aligned}
\sum_{\sigma \in Z^{n-m}} A_{\sigma} X^{\sigma}= & \sum_{\sigma_{1} \geqq 0, \cdots, \sigma_{n-m} \geqq 0} A_{\sigma} X^{\sigma} \\
& +\sum_{\sigma_{1}<0, \sigma_{2} \geqq 0, \cdots, \sigma_{n-m} \geqq 0} A_{\sigma} X^{\sigma} \\
& +\cdots+\sum_{\sigma_{1}<0, \cdots, \sigma_{n-m}<0} A_{\sigma} X^{\sigma} .
\end{aligned}
$$

Since we can write $r_{i}\left(x_{i}\right)^{\sigma_{i}}=r_{i}\left(-x_{i}\right)^{-\sigma_{i}}$ when $\sigma_{i}<0$, we obtain similar inequalities as (3.6) and each term in the right side of (3.7) is uniformly absolutely convergent in the wider sense on $\boldsymbol{C}^{n-m}$. Hence $\sum_{\sigma \in \boldsymbol{Z}^{n-m}} \boldsymbol{g}_{x, \sigma}$ is 
uniformly absolutely convergent in the wider sense on $C^{n-m}$. Let $G(x):=$ $g_{x}(0)$. Since each $g_{x, \sigma}$ is holomorphic, $G(X)$ is a holomorphic function on $C^{n-m}$. It follows from (3.5) that

$$
G\left(x+\gamma_{2}\right)-G(x)=a(\gamma, x)-a(\gamma, 0)+G\left(\gamma_{2}\right)
$$

for all $\gamma \in \Gamma$. This implies that a factor of automorphy $\alpha(\gamma, x)=\exp (\alpha(\gamma, x))$ is equivalent to a representation $\exp (\phi(\gamma))$ of $\Gamma$, where $\phi(\gamma):=a(\gamma, 0)-$ $G\left(\gamma_{2}\right)$.

\section{REFERENCES}

[1] Abe, Y., $(H, C)$-groups with positive line bundles, Nagoya Math. J., 107 (1987), 1-11. (in press)

[2] Cousin, P., Sur les fonctions triplement périodiques de deux variables, Acta Math., $1-11$.

[ 3 ] Kazama, H., $\bar{\partial}$ Cohomology of (H,C)-groups, Publ. R.I.M.S. Kyoto Univ., 20 (1984), 297-317.

[ 4 ] Kazama, H. and Umeno, T., Complex abelian Lie groups with finite-dimensional cohomology groups, J. Math. Soc. Japan, 36 (1984), 91-106.

[ 5 ] Kopfermann, K., Maximale Untergruppen Abelscher komplexer Liescher Gruppen, Schr. Math. Inst. Univ. Münster, 29 (1964).

[6] Malgrange, B., La cohomologie d'une variété analytique complexe à bord pseudoconvexe n'est pas nécessairement séparée, C.R. Acad. Sci. Paris Sér. A, 280 (1975), 93-95.

[ 7 ] Matsushima, Y., Fibrés holomorphes sur une tore complexe, Nagoya Math. J., 14 (1959), 1-14.

[8] Morimoto, A., Sur la classification des espaces fibrés vectories holomorphes sur une tore complexe admettant des connexions holomorphes, Nagoya Math. J., 15 (1959), 83-154.

[ 9 ] - Non-compact complex Lie groups without non-constant holomorphic functions, Proc. Conf. on Complex Analysis (Minneapolis 1964), Springer, 1965, 256-272.

[10] - On the classification of non-compact complex abelian Lie groups, Trans. Amer. Math. Soc., 123 (1966), 200-228.

[11] Vogt, Ch., Line bundles on toroidal groups, J. Reine Angew. Math., 335 (1982), $197-215$.

[12] —- Two remarks concerning toroidal groups, Manuscripta Math., 41 (1983), 217-232.

Department of Mathematics

Toyama University

Gofuku, Toyama 930, Japan 